\title{
Clinical and Epidemiological Features and Management of Neonatal Hypoglycemia at the University Teaching Hospital of Treichville (Abidjan-Côte d'Ivoire)
}

\author{
Gneneyeri Joseph Ouattara ${ }^{*}$, Lassina Cissé ${ }^{1}$, Gervais Koffi², Jean-Jacques Atteby Yao ${ }^{1}$, \\ Jacob Enoh ${ }^{1}$, Corinne Sei ${ }^{1}$, Soumahoro Oulai ${ }^{1}$ \\ ${ }^{1}$ Department of Paediatrics, University Teaching Hospital of Treichville, Abidjan, Côte d'Ivoire \\ ${ }^{2}$ Unit of Biochemistry, Central Laboratory, University Teaching Hospital of Treichville, Abidjan, Cote d'Ivoire \\ Email: *jgouattara@yahoo.fr
}

How to cite this paper: Ouattara, G.J., Cissé, L., Koffi, G., Yao, J.-J.A., Enoh, J., Sei, C. and Oulai, S. (2017) Clinical and Epidemiological Features and Management of Neonatal Hypoglycemia at the University Teaching Hospital of Treichville (Abidjan-Côte d'Ivoire). Open Journal of Pediatrics, 7, 320-330.

https://doi.org/10.4236/ojped.2017.74037

Received: October 11, 2017

Accepted: December 9, 2017

Published: December 12, 2017

Copyright $\odot 2017$ by authors and Scientific Research Publishing Inc. This work is licensed under the Creative Commons Attribution International License (CC BY 4.0).

http://creativecommons.org/licenses/by/4.0/

\begin{abstract}
The purpose of this study is to determine the prevalence, the characteristics of hypoglycaemia in the newborn admitted to emergency departments of teaching Hospital of Treichville, Abidjan and to evaluate the relevance of capillary blood glucose in this diagnosis. Patients and Methods: This prospective cross-sectional study was held from 01 February to 25 June 2017 in the Pediatric Emergency Department. It involved 201 newborns under 24 hours of age who consulted in pediatric emergency for any reason. Data were collected from parent direct interviews, analysis of the follow-up record, physical examination of the newborn, results of glucose level in the capillary blood at heel and in venous blood. On dry tube and gray tube (containing sodium fluoride and potassium oxalate), the blood glucose on the latter tube constituting the reference blood glucose, with neonatal hypoglycaemia defined by a venous blood glucose less than or equal to $2.5 \mathrm{mmol} / \mathrm{l}(0.45 \mathrm{~g} / \mathrm{l})$. The statistical tests used were the frequency comparison, the Chi Square, the Fisher Test with a significance threshold $\mathrm{p}<$ 0.05 or the Odds-ratio with a confidence interval of $95 \%$ and a significance threshold of 5\%, but also with the correlation coefficient. Results: Newborns of less than 3 hours of life (70.6\%) were the most represented with a male predominance (sex ratio $=2.9$ ). The reasons for consultation were dominated by neurological disorders (36.5\%), prematurity (31.8\%), and fever $(22.2 \%)$. The average time to complete the blood glucose test was less than one hour after the sampling in $6.5 \%$ of the cases. The mean blood glucose was $0.59 \mathrm{~g} / \mathrm{l}(3.28 \mathrm{mmol} / \mathrm{l})$ with extremes ranging from 0.15 to $1.8 \mathrm{~g} / 1(0.83 \mathrm{mmol} / \mathrm{l}$ to $10 \mathrm{mmol} / \mathrm{l})$. The prevalence of hypoglycemia by the instantaneous glycemic method was $15.9 \%$.
\end{abstract}


On tube containing sodium fluoride and potassium oxalate, it was $20.4 \%$, while the prevalence of red blood cell hypoglycemia was $38.1 \%$. A good correlation was found between capillary glycemia and gray tube with $r=0.97$. Signs associated with hypoglycemia were predominantly male $(\mathrm{p}<0.05)$, prematurity ( $\mathrm{p}$ $<0.05$ ). As for cerebral suffering, it had proved to be a protective factor (Fisher: 0.002 ). The mortality rate was $2.5 \%$. Hypoglycemia was not found. Conclusion: Hypoglycaemia is relatively common in our working setting, particularly in premature infants. However, it should be carefully sought in any newborn admitted to emergency regardless of the reason for admission. The use of capillary blood glucose proves to be a good alternative because it allows a fast management.

\section{Keywords}

Hypoglycaemia, Newborn, Prevalence, Emergency

\section{Introduction}

According to the American Academy Pediatric, neonatal hypoglycaemia is defined as a venous blood glucose lower than or equal to $2.5 \mathrm{mmol} / \mathrm{l}(0.45 \mathrm{~g} / \mathrm{l})$ [1]. The hypoglycaemia of the newborn is a real emergency and its correction must be fast. At birth, there is a physiological change in the hormone profile of the newborn with increased stress hormones associated with a fall in insulin levels. This leads to a cascade of events that maintain glycemic control [2]. In some situations, this carbohydrate balance can be disrupted, which is at the origin of hypoglycaemia. The threshold for pathological neonatal hypoglycemia is variable according to different studies [3]. This blood glucose can be influenced by many factors, including prematurity, gestational diabetes, birth condition, other pathologies or situations of the newborn and even of the conditions of withdrawal [4] and hence, the difficulty of having a prevalence of neonatal hypoglycemia. The dosage of neonatal blood glucose is not systematic [5]. It must be sought before risk factors which are well systematized [6]. However, in the absence of these risk factors, hypoglycaemia can often be noted and is very often rapidly corrected and without sequelae. We speak of transient hypoglycemia. The diagnosis of significant neonatal hypoglycemia is based on the existence of an evocative clinical picture and biological hypoglycaemia (venous blood glucose). It is established in the newborn that the most common strategy used to reduce morbidity and the risk of neurodevelopmental sequelae and hypoglycaemia-related mortality is routine screening for any newborn who goes badly [7]. The acquisition and availability of capillary blood glucose monitors could be a viable alternative to this strategy. It is clear from our observations in the newborn care services, particularly in Abidjan, that few teams regularly have capillary blood glucose meters (at the emergency room). Therefore, hypoglycaemia would not be promptly sought in newborns who are ill at emergencies. Moreover, what degree of relia- 
bility of the apparatus is used for the circumstance? The purpose of this study is to determine the prevalence of hypoglycaemia in newborns admitted to the Treichville $\mathrm{CHU}$ at an emergency, to describe their epidemiological and clinical characteristics, and to situate capillary glycemia (blood glucose) in the strategy diagnostic.

\section{Methodology}

1) Study Framework

The study was conducted at the Treichville University Hospital in Abidjan, Côte d'Ivoire. It is a 3-levelhospital of the health pyramid in Côte d'Ivoire. The Pediatric Department has a unit for the care of newborns. This unit is the only public hospitalization structure for newborns in the southern district of Abidjan. It has a capacity of 35 places and is equipped with a sub-unit for the care of premature babies (incubators), a 6-seater kangaroo sub-unit, and non-ventilation equipment invasive. The study was therefore carried out in pediatric emergency rooms, the main gateway for newborns admitted to pediatrics.

2) Type of Study

It is a prospective, transversal, and analytical study which was conducted from 01 February to 25 June 2017.

3) Study Population

It included newborns admitted at the Emergency Department of the Pediatric Ward.

The main inclusion criteria were to be aged no more than 24 hours at admission, not to have received glucose serum perfusion within the hour prior to admission In addition, parents should have given their consent to participate in the study.

4) Sampling

The required sample size was calculated according to formula [8]: $n=\varepsilon^{2} P(1-P) / i^{2}$, with $i=5 \%, \varepsilon=1.96, \mathrm{P}=15 \%$ which made 196 patients. The sample was obtained by systematic sampling with a sampling interval of $15(\mathrm{~N} / \mathrm{n}$, where $\mathrm{N}$ was the size of study population 2208 and $\mathrm{n}$ was the sample size) on the list of newborns received at emergencies in 2017.

The blood was collected by the usual method of sampling (in the department) after cleaning under usual aseptic conditions these samples were made in two different tubes: one tube containing sodium fluoride and potassium oxalate (vacutainer $^{\circledR}$ gray tube), a dry tube (vacutainer ${ }^{\circledR}$ red tube) and blood glucose (heel) was made at the time of sampling. The glucose oxidase-peroxidase enzyme was assayed by centrifugation on a multi-parametric automaton: (Randox-Monaco) to the Biochemistry Unit of the Central Laboratory of the Treichville UHC from 8 am to $4 \mathrm{pm}$.

The diagnosis of hypoglycaemia was made before a venous blood glucose containing sodium fluoride of less than $0.45 \mathrm{~g} / \mathrm{l}(2.5 \mathrm{mmol} / \mathrm{l})$.

When hypoglycaemia was established, surveillance was required. The blood glu- 
cose was checked every 2 or 3 hours by the capillary method until the hypoglycemia was corrected.

The data collection of the survey was based on the direct interview with the newborns' parents, the follow-up record, the physical examination, and the results of the different glucose. Newborns who met the inclusion criteria were 201.

5) Statistical Analysis

The statistical tests used were the frequency comparison, the Chi Square Test the Fisher Test with a significance threshold $\mathrm{p}<0.05$ or the Odds-ratio with a confidence interval of $95 \%$ and a significance threshold of $5 \%$, but also with the correlation coefficient.

- We had the agreement with the medical and scientific committee of the University of Teaching Hospital of Treichville before this study taken place. The anonymous of the process has strictly observed.

\section{Results}

There was a male predominance $($ sex ratio $=2.9$ ). Newborns under 3 hours of intake were the most represented $(70.6 \%)$, and the mean age at admission was 2.5 hours (0.34 - 23 hours).

The main reasons for admission to emergencies were prematurity (31.8\%) and neurological disorders consisting of convulsions and neonatal distress (36.5\%) (Table 1)

The mean weight of newborns included in the study was 2850 grams \pm 0.625 (1100 g - $4450 \mathrm{~g})$.

Clinical manifestations were dominated by prematurity (31.8\%), cerebral suffering according to the clinical classification of SARNAT [9] (25.4\%) and respiratory distress $(20.6 \%)$. The estimate of the mean time to analysis of blood samples was 1.45 hours and that only $6.5 \%$ of samples were analyzed less than one hour after collection, $82.5 \%$ analyzed between 1 hour and 2 hours and $11 \%$ after 2 hours (Table 2).

The prevalence of hypoglycaemia was $20.4 \%$ on the tubes containing sodium fluoride and potassium oxalate (reference glucose). The results of capillary and dry tube glucose were shown in Table 2. The mean blood glucose was $0.59 \mathrm{~g} / 1$ $(3.28 \mathrm{mmol} /)$.

Signs associated with hypoglycaemia (Table 3 ) were male $(\mathrm{OR}=5.2, \mathrm{p}<$ $0.05)$, prematurity $(\mathrm{OR}=3.3, \mathrm{p}<0.05)$, and neurological distress be a protective sign against hypoglycaemia $(\mathrm{F}=0.002$ and $\mathrm{p}<0.05)$. Moreover, the age of the newborn at admission and the time at which the blood glucose was achieved at the laboratory had no influence on the occurrence of hypoglycaemia (OR 0.76 $[0.33-1,78] \mathrm{p}=0.54)$.

Gray tube venous blood glucose levels were strongly correlated with capillary blood glucose levels $(r=0.97)$ (Figure 1 ), while gray tube venous blood glucose levels were fairly correlated with venous blood glucose levels in the dry tube $(\mathrm{r}=$ 0.55) (Figure 2). 
The hypoglycaemias were rapidly corrected (less than 24 hours after admission) by the usual management protocol (hypertonic solution or dextrin Maltose).

Immediate evolution was marked by the occurrence of a death in $2.5 \%$ of cases. However, the lethality linked to hypoglycemia was zero. Indeed, the newborns who were seriously ill had benefited from regular glycemic control (2 to 3 hours).

Table 1. Distribution by evacuation reasons.

\begin{tabular}{ccc}
\hline Reason of evacuation & Number & Percentage \\
\hline Prematurity & 64 & 31.8 \\
Convulsion & 19 & 9.5 \\
Fever & 45 & 22.2 \\
Neonatal suffering & 54 & 27 \\
Icterus & 6 & 3.2 \\
Macrosomia & 10 & 5 \\
\hline
\end{tabular}

Table 2. Hypoglycemic numbers according to the time required for the laboratory examination.

\begin{tabular}{|c|c|c|c|c|c|}
\hline & \multirow[t]{2}{*}{ Blood glucose time limit } & {$[0-1$ hour [} & $\begin{array}{l}{[1 \mathrm{~h}-2} \\
\text { hours [ }\end{array}$ & $>2$ hours & \multirow[t]{2}{*}{ total } \\
\hline & & \multicolumn{3}{|c|}{ Number } & \\
\hline & hypoglycemia & 32 & - & - & \multirow{2}{*}{$\begin{array}{c}32 \\
169\end{array}$} \\
\hline & Blood glucose $>0.45 \mathrm{~g} / \mathrm{l}(2.5 \mathrm{mmol} / \mathrm{l})$ & 169 & - & - & \\
\hline \multirow{2}{*}{$\begin{array}{l}\text { Blood glucose on } \\
\text { gray tube }\end{array}$} & hypoglycemia & 0 & 38 & 3 & 41 \\
\hline & Blood glucose $>0.45 \mathrm{~g} / \mathrm{l}(2.5 \mathrm{mmol} / \mathrm{l})$ & 13 & 128 & 19 & 160 \\
\hline \multirow{2}{*}{$\begin{array}{c}\text { Blood glucose on } \\
\text { dry tube }\end{array}$} & hypoglycemia & 3 & 63 & 10 & 76 \\
\hline & Blood glucose $>0.45 \mathrm{~g} / \mathrm{l}(2.5 \mathrm{mmol} / \mathrm{l})$ & 10 & 103 & 12 & 125 \\
\hline
\end{tabular}

Table 3. Hypoglycaemia by different signs.

\begin{tabular}{ccc}
\hline & Number of hypoglycaemia & $\mathrm{p}$ \\
\hline Males & 35 & $(\mathrm{p}<0.05)$ \\
Prematurity & 22 & $(\mathrm{p}<0.05)$ \\
Convulsion & 1 & - \\
Fever & 10 & $(\mathrm{p}=0.61)$ \\
Neonatal suffering & 3 & $(\mathrm{p}<0.05)$ \\
Respiratory distress & 13 & $(\mathrm{p}=0.25)$ \\
Icterus & 0 & $(\mathrm{p}=0.43)$ \\
Macrosomia & 3 & $(\mathrm{p}=0.54)$ \\
Hour of life $<3$ hours & 36 &
\end{tabular}




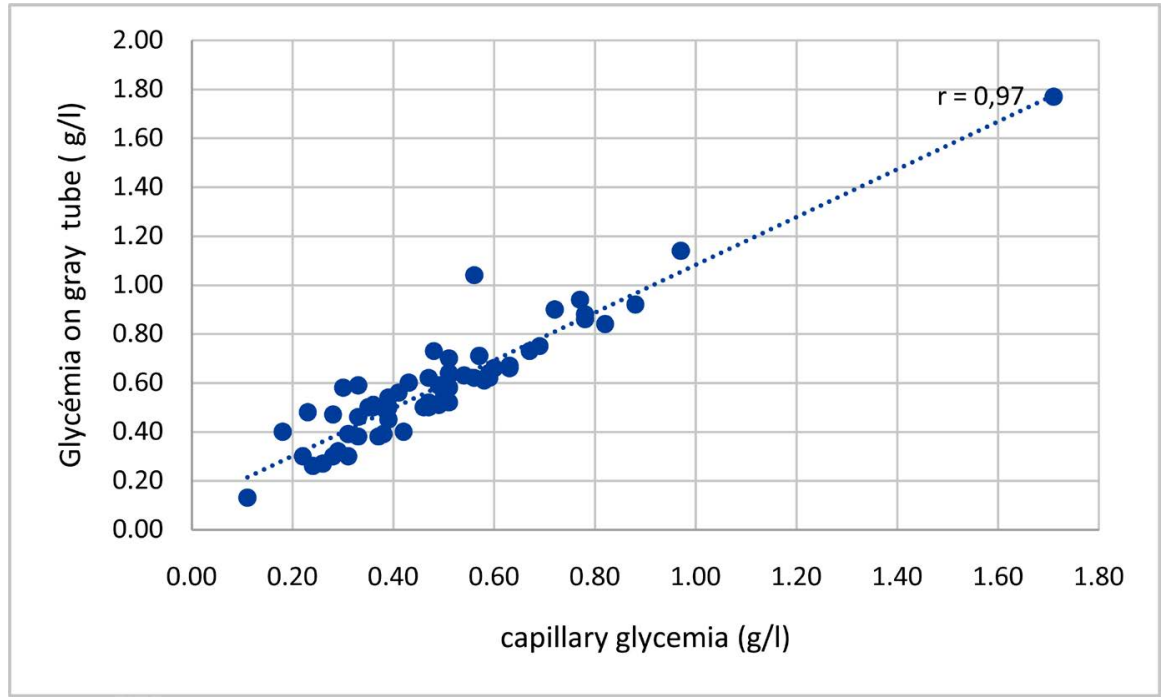

Figure 1. Correlation between capillary glycemia and blood glucose measured on gray from laboratory.

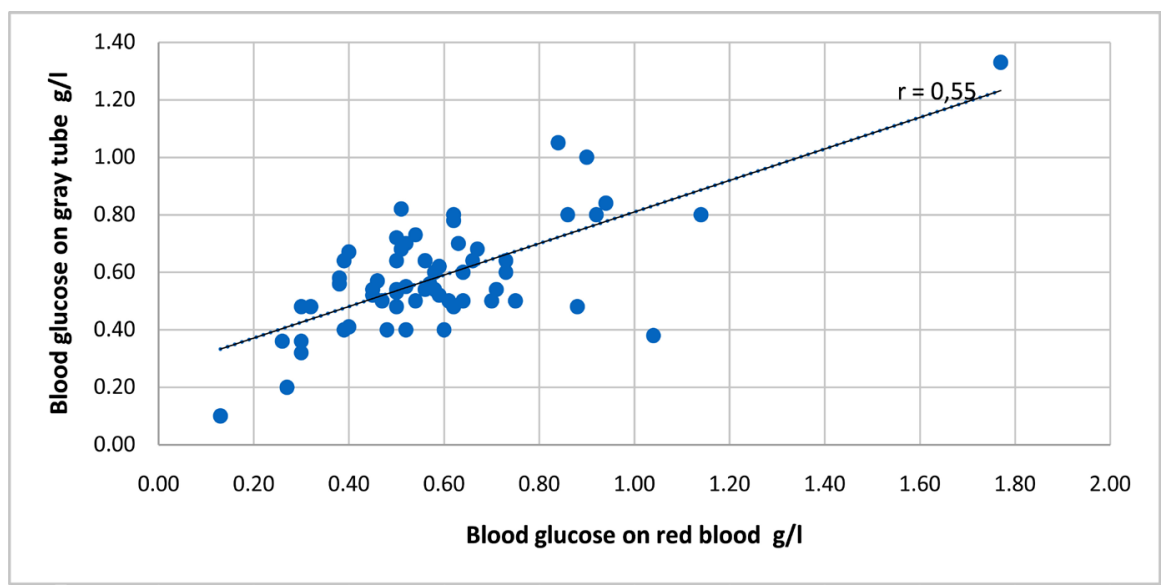

Figure 2. Correlation between blood glucose measured on gray tube (reference) and dry blood.

\section{Discussion}

In Canada, the prevalence of neonatal hypoglycaemia was 12\% - 14\% [10] and $11.4 \%$ in Mali [11], which is approximately identical to that of Houenou in 2000 at the Cocody Hospital in Abidjan [12]. These results are slightly lower than our findings. And could be due that the blood glucose level was sampled in newborns in maternity for prevalence in Canada. Moreover, in the studies of Keita in Mali and Gneba in Côte d'Ivoire, newborns were already hospitalized in neonatology, whereas in our study they had just been admitted. The newborns included had less than 24 hours of life, as in the study done by Amponsah et al. [13] in Ghana, but in the latter the prevalence was only $1.6 \%$ for a dosage systematic glucose levels in all newborns included in their study. As noted in several studies and recommendations, including those of the Canadian Paediatric Society, hypoglycaemia must be rapidly sought in vulnerable term infants; including those with acute 
illness or those whose mother is diabetic or exhibits macrosomia or growth retardation [10]. In our study, all neonates with hypoglycaemia (20.6\%) had been received for a particular reason.

Furthermore, as in other authors (Gneba et al. and Keita), a male predominance was found. The susceptibility of male infants to different pathologies may explain this trend.

In our study, hypoglycaemia was associated with male sex, prematurity. As for neurological disorders, they appeared to be a protective factor against hypoglycaemia. The difficulty would be whether hypoglycaemia was responsible for clinical manifestations or whether it was one of the consequences of the clinical picture presented by these newborns. For Cornblath et al. [14] "significant hypoglycaemia is not and cannot be defined as a single figure that could be universally attributed to any patient. It is characterized rather by a value specific to each individual and varies with its state of physiological maturity and the influence of pathology".

Indeed, the clinical picture is rarely evocative. The signs or the specific expression of neonatal hypoglycaemia are most often unidentifiable. Signs and symptoms are very often absent despite blood glucose lower than the values considered as lower threshold values. In rare cases where these clinical manifestations are very expressive, these manifestations are dominated by convulsions. Clinical manifestations are highly variable and non-specific. In our study, we found that hypoglycaemia was related to the clinical picture. Thus, prematurity $(\mathrm{p}=0.002)$ and the male genus were related to hypoglycaemia, whereas brain suffering $($ Fisher $=0.002)$ appeared to be a protective factor against hypoglycaemia, reported by Pam et al, where $65 \%$ of the low birth weight had hypoglycaemia [15]. Clinically, significant neonatal hypoglycaemia is an important health problem, which could have implications for neurodevelopment [16]. Prolonged or extremely severe hypoglycaemia is associated with an uncertain prognosis, while symptomatic hypoglycaemia is linked to a series of brain lesions showed by Magnetic Resonance Imaging [17]. It is clear that hypoglycaemia must be actively investigated and corrected. Moreover, the factors which increase the risk of hypoglycaemic brain damage are still poorly understood. However, studies indicate that some newborns, particularly those with hyperinsulinemia, cannot access other substrates of brain energy during the first few days of life, while milk consumption is low. The prognosis of asymptomatic hypoglycaemia does not necessarily differ from that of symptomatic hypoglycaemia if the convulsions of the "symptomatic" group are removed [18]. Conversely, routine screening for hypoglycaemia in at-risk children may have iatrogenic effects in that it is well established that stress or repeated pain are also factors that may inhibit brain development in premature babies [19].

In the literature, it is reported that $50 \%$ of hypoglycaemic convulsions cause sequelae, in the longer term. These sequelae are dominated by severe cortical involvement with predominant parieto-occipital regions. Cortical atrophy with clinical consequences marked by disturbed neuro-motor and cognitive scores [20] can 
also be noted.

Surveillance recommendations, such as those used by the Canadian Paediatric Society (CPS) for early detection of hypoglycaemia [5] [10]. Hypoglycaemia should be sought in the face of risk factors that are well systematized [6]. As risk factors, the clinical condition of the newborn, this explains the choice of the dosage of blood glucose in newborns admitted to paediatric emergencies.

In neonates, the presence of neurological disorders, respiratory disorders or a clear neonatal infection should not obscure the possibility of associated neonatal hypoglycaemia. Our findings were therefore in favour of this hypothesis where all our hypoglycaemias were associated with other pathologies, and this hypoglycaemia had been corrected less than 24 hours after admission. They were transient.

The diagnosis is usually made before a respiratory or even neurological emergency. In emergency, capillary blood glucose is important although the diagnosis of certainty is made by laboratory tests (venous blood glucose) [6]. The challenge would be to have blood glucose results made in the laboratory in a short time in our conditions of practice, which is still very difficult in view of our results where less than one tenth (6.5\% of cases) blood glucose results were obtained less than one hour after sampling. The realization of capillary blood glucose would be an alternative of choice awaiting retrospective confirmation on venous blood glucose as claimed by Léger [21].

It should also be noted that the glycaemia obtained by capillary blood glucose was given with margins of error [22] [23]. The difficulty in using this latter technique would come from the regular calibration, the sensitivity of the strips, the disparity among the manufacturers, and the sensitivity of the capillary glucose meter. Although hypoglycaemia is much less common in newborns who do not have predetermined risk factors, this small group may be particularly vulnerable to complications due to delayed diagnosis and treatment. Moreover, in the majority of cases of neonatal hypoglycaemia, this is a transient hypoglycaemia that is spontaneously corrected and does not appear to be deleterious. It is therefore inappropriate to systematically monitor blood glucose in all newborns [24], but to be aware of the possibility of hypoglycaemia associated with any abnormal signs in the newborn, and so to dose capillary blood glucose urgently in such a context.

In the present study, hypoglycaemia using the capillary blood glucose technique (test strips) was found in $15.9 \%$ of patients, slightly below venous blood glucose levels containing sodium fluoride (20.6\%). Capillary blood glucose is an element of strong presumption of hypoglycaemia and allows a prompt management of hypoglycaemia. It is also important to note that all lower capillary glycaemias were confirmed by the reference blood glucose in our study.

In addition, blood glucose measurements using dipsticks are not sufficiently reliable to establish the diagnosis [25]; however, in our study, there was a strong correlation between capillary glycaemia and baseline blood glucose $(r=0.97)$. This find- 
ing was also made by Djohan et al. [26] in a study conducted in Côte d'Ivoire, where he noted a strong correlation between venous blood glucose (cord blood) and capillary blood glucose with correlation factor $(r=0.98)$. Thus, capillary blood glucose could be a useful tool for screening neonatal hypoglycaemia which should be confirmed by venous blood glucose without delaying its management.

It is certain that the measurement of blood sugar alone cannot provide all the necessary information to fully appreciate the cerebral energy situation. Indeed, the clinical importance of hypoglycaemia depends on several factors, such as the depth of hypoglycaemia, the frequency, and duration of episodes, and possible compensations by alternative substrates, such as ketones and lactate. However, we cannot routinely evaluate the availability of all alternative fuels, and we only have discontinuous information. In addition, there are problems of interpretation related to the site of collection (venous or capillary peripheral blood is subjected to insulin), the type of blood on which the measurement is made (blood glucose levels are lower in whole blood than in plasma) and the measurement method [1].

In any event, rapid treatment should shorten the period of hypoglycaemia and symptoms and, as a result, reduce the risk of complications [27], as reported by Burns in 2008 [28]. Neonates with prolonged hypoglycaemia experienced neurological abnormalities in Magnetic Resonance Imaging.

\section{Conclusion}

Neonatal hypoglycaemia, which is relatively frequent in our conditions, must be actively sought in any newborn or with evocative symptoms by the use of capillary blood glucose (test strips). This is the best strategy to reduce the morbidity associated with hypoglycaemia, and therefore, prevent neurological sequelae or even deaths related to this hypoglycaemia.

\section{References}

[1] David, H., Adamkin, M.D. and Committee on Fetus and Newborn (2011) Clinical Report-Postnatal Glucose Homeostasis in Late-Preterm and Term Infants. Pediatrics, 127, 575-579. https://doi.org/10.1542/peds.2010-3851

[2] Mitanchez, D. (2008) Ontogenesis of Glycemic Regulation and Consequences for the Management of the Newborn. Archives de Pédiatrie, 15, 64-74. https://doi.org/10.1016/j.arcped.2007.10.006

[3] Cornblath, M., Hawdon, J.M., Williams, A.F., et al. (2000) Controversies Regarding Definition of Neonatal Hypoglycemia: Suggested Operational Thresholds. Pediatrics, 105, 1141-1145. https://doi.org/10.1542/peds.105.5.1141

[4] Jane, M. (2014) HawdonNeonatal Hypoglycemia: Are Evidence-Based Clinical Guidelines Achievable? NeoReviews, 15, e91. https://doi.org/10.1542/neo.15-3-e91

[5] Touati, G. (2005) Hypoglycemia in Children. Metabolismes Diabetes Hormones and Nutrition (IX), No. 5, 164-166.

[6] Francoual, C., Bouillié, J., Parat-Lesbros, S. (2008) The Newborn in the Birth Room. Pediatrics Maternity, 33, 418-421. 
[7] Thompson-Branch, A. and Havranek, T. (2017) Neonatal Hypoglycemia. Pediatrics in Review, 38, 147. https://doi.org/10.1542/pir.2016-0063

[8] Lwaga, S.K. and Lemeshow, S. (1991) Sample Size Determination in Health Studies: A Practical Manual. World Health Organization, Geneva, 62.

[9] Sarnat, H.B. and Sarnat, M.S. (1976) Neonatal Encephalopathy. Following Foetal Distress. A Clinical and Electroencephalography Study. Archives of Neurology, 33, 696-705. https://doi.org/10.1001/archneur.1976.00500100030012

[10] Aziz, K. and Dancey, P. (2004) Canadian Pediatric Society, Fetal and Newborn Study Committee (Statement of Principles). Guidelines for the Screening of Newborns Vulnerable to Hypoglycemia. Paediatrics \& Child Health, 9, 733-740.

[11] Keita, O. (2000) Evaluation of Glycemic Disorders in Early Neonatal Period. Master Thesis, Bamako University, Mali.

[12] Gneba, C. (2000) Prevalence and Risk Factors of the Neonatal Hypoglycemia in the University of Teaching Hospital of Cocody. Master Thesis, Felix Houphouet Boigny University, Abidjan.

[13] Amponsah, G., Hagan, O.C.K. and Okai, E. (2015) Neonatal Hypoglycaemia at Cape Coast Teaching Hospital. Journal of the West African College of Surgeons, 5, 100-116.

[14] Cornblath, M. and Ichord, R. (2000) Hypoglycemia in the Neonate. Seminars in Perinatology, 24, 136-149. https://doi.org/10.1053/sp.2000.6364

[15] Pam, S., Ejeliogu, E., Pam, V., Madziga, I., Collins, J., Toma, B. and Adekwu, O. (2006) Hypoglycaemia in Fasting Low Birth Weight Infants in Jos. Nigeria Annals of African Medicine, 5, 46-51.

[16] Boardman, J.P., Wusthoff, C.J. and Cowan, F.M. (2013) Hypoglycaemia and Neonatal Brain Injury. Archives of Disease in Childhood. Education and Practice Edition, 98, 2-6. https://doi.org/10.1136/archdischild-2012-302569

[17] Alkalay, A.L., Flores-Sarnat, L., Sarnat, H.B., Moser, F.G. and Simmons, C.F. (2005) Brain Imaging Findings in Neonatal Hypoglycemia: Case Report and Review of 23 Cases. Clinical Pediatrics, 44, 783-790. https://doi.org/10.1177/000992280504400906

[18] Koivisto, M., Blanco-Sequeiros, M. and Krause, U. (1972) Neonatal Symptomatic and Asymptomatic Hypoglycaemia: A Follow-Up Study of 151 Children. Developmental Medicine \& Child Neurology, 14, 603-614. https://doi.org/10.1111/j.1469-8749.1972.tb02642.x

[19] Grunau, R.E., Holsti, L. and Peters, J.W. (2006) Long-Term Consequences of Pain in Human Neonates. Seminars in Fetal and Neonatal Medicine, 11, 268-275. https://doi.org/10.1016/j.siny.2006.02.007

[20] Boluyt, N., et al. (2006) Neurodevelopment after Neonatal Hypoglycemia. Pediatrics, 117, 2231-2243.

[21] Bourillon, A. and Leger, J. (2008) Endocrinologie et diabète. In: Pediatrics for the Practitioner, 5th Edition, Vol. 13, 290-294.

[22] Srinivasan, G., Pildes, R.S., Cattamanchi, G., Voora, S. and Lilien, L.D. (1986) Plasma Glucose Values in Normal Neonates: A New Look. Journal of Pediatrics, 109, 114-117. https://doi.org/10.1016/S0022-3476(86)80588-1

[23] Nicholl, R. (2003) What Is the Normal Range of Blood Glucose Concentrations in Healthy Term Newborns? Archives of Disease in Childhood, 88, 238-239. https://doi.org/10.1136/adc.88.3.238

[24] Hussain, K. and Sharief, N. (2000) The Inaccuracy of Venous and Capillary Blood 
Glucose Measurement using Reagent Strips in the Newborn Period and the Effect of Haematocrit. Early Human Development, 57, 111-121. https://doi.org/10.1016/S0378-3782(99)00060-2

[25] Marcus, C. (2001) How to Measure and Interpret Glucose in Neonates. Acta Paediatrica, 90, 963-964. https://doi.org/10.1111/j.1651-2227.2001.tb01348.x

[26] Djohan, Y.F., Cisse-Camara, M., Niamke, A.G., Koffi, G., Dere, L., Monde, A.A., Tiahou, G., Djessou, P. and Sess, D. (2015) Interest of Venous Umbilical Cord Blood in the Diagnosis of Neonatal Hypoglycemia. International Journal of Biological and Chemical Sciences, 9, 2574-2581. https://doi.org/10.4314/ijbcs.v9i5.26

[27] Rozance, P.J. and Hay, W.W. (2006) Hypoglycemia in Newborn Infants: Features Associated with Adverse Outcomes. Biology of the Neonate, 90, 74-86. https://doi.org/10.1159/000091948

[28] Burns, C.M., Rutherford, M.A., Boardman, J.P., et al. (2008) Patterns of Cerebral Injury and Neurodevelopmental Outcomes after Symptomatic Neonatal Hypoglycemia. Pediatrics, 122, 65-74. https://doi.org/10.1542/peds.2007-2822 